
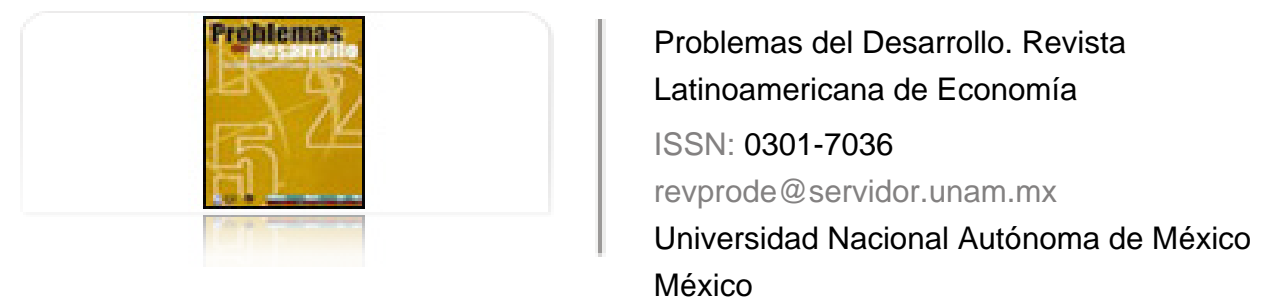

Fuentes, Noé Arón; García Andrés, Adelaido JERARQUIZACIÓN SECTORIAL DE LA ECONOMÍA MEXICANA: UN ENFOQUE DE TEORÍA DE GRAFOS

Problemas del Desarrollo. Revista Latinoamericana de Economía, vol. 40, núm. 158, julio-septiembre, 2009, pp. 137-159

Universidad Nacional Autónoma de México

Distrito Federal, México

Disponible en: http://www.redalyc.org/articulo.oa?id=11820200006

Cómo citar el artículo

- Número completo

- Más información del artículo

Página de la revista en redalyc.org

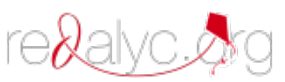

Sistema de Información Científica

Red de Revistas Científicas de América Latina, el Caribe, España y Portugal Proyecto académico sin fines de lucro, desarrollado bajo la iniciativa de acceso abierto 


\title{
JERARQUIZACIÓN SECTORIAL DE LA ECONOMÍA MEXICANA: UN ENFOQUE DE TEORÍA DE GRAFOS
}

\author{
Noé Arón Fuentes* \\ Adelaido García Andrés**
}

Fecha de recepción: 13 de abril de 2009. Fecha de aceptación: 29 de julio de 2009.

\section{Resumen}

En este estudio se aplica la teoría de grafos para realizar un análisis estructural de la economía mexicana; la fuente de información es la matriz de transacciones intersectoriales de la tabla nacional de insumo-producto, 2003 (INEGI, 2008).

El grafo de influencia relativa asociado a la tabla nacional permite analizar las relaciones de dominación-dependencia entre los diferentes sectores productivos en su doble vertiente de compradores y vendedores, lo que también brinda una visión estructural del comportamiento del conjunto de tales relaciones.

El método permite realizar una jerarquización sectorial que tiene en cuenta las relaciones globales de dependencia; se basa en unas medidas relativas de atracción que, al hacer abstracción del peso individual de cada sector de la economía, proporcionan una visión más real de la estructura de las relaciones intersectoriales. Cabe destacar que éste es un aspecto no incorporado en el análisis estructural clásico de insumo-producto.

Palabras clave: insumo-producto, teoría de grafos, análisis estructural.

Clasificación JEL: C67, D57, L60

* Director del Departamento de Estudios Económicos de El Colegio de la Frontera Norte y SNI nivel III. correo electrónico: afuentes@ colef.mx

** Estudiante de la Maestría en Economía Aplicada de El Colegio de la Frontera Norte. correo electrónico: adelgaran@ colef.mx 


\section{Abstract}

In this study we applied the graphic theory in order to make a structural analysis of the Mexican economy, using as basic information the matrix of intersectorial transactions of the national input-output table, 2003 (INEGI, 2008).

By analyzing the graphic of relative influence associated to the national table, we can study the "domination-dependence" relationship existing between productive sectors, both in their buying and selling aspect, achieving at the same time a structural vision of the joint behavior of these relationships.

The method allows a sectorial hierarchy considering the global dependence relationships on the basis of relative measures of attraction, by ignoring the individual weight of each sector in the economy, yield a realistic vision of its intersectorial relationships. Finally, this is an aspect not incorporated in the classical input output structural analysis.

Key Words: Input Output, Graphic Theory and Structural Analysis

Classification JEL: C67, D57, L60

\section{Résumé}

Dans cette étude est appliquée la Théorie des Graphes pour réaliser une analyse structurelle de l'économie mexicaine, en prenant comme information la matrice de transactions intersectorielles de la table nationale de produit-facteur de production, 2003 (INEGI, 2008).

En analysant le graphe d'influence relative associée à la table nationale, il est possible d'analyser les relations de "domination-dépendance" qui existent entre les différents secteurs productifs dans leur double figure d'acheteurs et de vendeurs, et d'obtenir ainsi dans le même temps une vision structurelle du comportement de l'ensemble de telles relations.

La méthode permet de réaliser une hiérarchisation sectorielle qui prend en compte les relations globales de dépendance sur la base de mesures relatives d'attraction qui, en faisant abstraction du poids individuel de chaque secteur de l'économie, fournissent une vision plus réelle de la structure des relations intersectorielles. Finalement, cet aspect n'est pas pris en compte dans l'analyse structurel classique de produit-facteur de production. Mots clés: produit-facteur de production, théorie des Graphes et analyse structurelle.

\section{Resumo}

Neste estudo aplica-se a Teoria de Grafos para realizar uma análise estrutural da economia mexicana, tomando como informação a matriz de transações intersetoriais da tabela nacional de insumo produto, 2003 (INEGI, 2008).

Analisando o grafo de influência relativa associada à tabela nacional podem-se analisar as relações de "dominação-dependência" existentes entre os diferentes setores produtivos em sua dupla vertente de compradores e vendedores, conseguindo ao mesmo tempo uma visão estrutural do comportamento do conjunto de tais relações.

$O$ método permite realizar uma hierarquia setorial que leva em conta as relações globais de dependência baseado em medidas relativas de atração que, ao abstrair-se do peso individual de cada setor da economia, proporcionam uma visão mais real da estrutura das relações intersetoriais. Finalmente, este é um aspecto não incorporado à análise estrutural clássica de insumo produto.

Palavras-chave: Insumo Produto, Teoria de Grafos e Análise Estrutural. 


\section{Introducción}

1 modelo de insumo-producto es una herramienta que permite evaluar las in-
terrelaciones e interdependencias entre los diferentes sectores productivos de
una economía. Hay algunos sectores productivos que se consideran más importantes porque asumen un mayor peso en el conjunto de las relaciones puestas en juego. Partiendo de esta idea, se han desarrollado mediciones del peso económico de cada sector dentro de la matriz de intercambios que permiten clasificar a los mismos en una tabla de insumo-producto. Esta clasificación posibilita identificar los sectores clave en toda la economía, así como los impulsores, los estratégicos y aquellos que son independientes.

Recientemente se ha desarrollado un novedoso enfoque de clasificación sectorial de la estructura productiva, basado en la teoría de grafos. Esta teoría tiene un gran potencial pues integra en su desarrollo cuestiones relevantes como la importancia de las interrelaciones sectoriales, que permiten calibrar las posiciones relativas entre sectores, su orientación o los caminos por donde circula la influencia económica dentro de la estructura considerada ${ }^{1}$. Por lo tanto, permite la detección de aquellos sectores clave para la economía en función de la importancia de las influencias productivas dentro de la estructura de intercambios.

El enfoque de grafos constituye una alternativa complementaria al enfoque clásico para el análisis de la tipificación sectorial de la economía, basado en la cuantificación de las relaciones directas entre los sectores a partir de la matriz de coeficientes técnicos (distribución) o de aquellos, basado en las relaciones globales sustentada en la matriz inversa de Leontief (inversa de distribución) porque, una debilidad importante del enfoque clásico es su dificultad para comprender qué ocurre con la capacidad relativa de cada sector económico para influir en otras actividades.

El propósito del presente trabajo es realizar una aplicación de la teoría de grafos al estudio de la estructura productiva mexicana usando la matriz de insumo producto nacional de 2003 (INEGI, 2008), ${ }^{2}$ realizando una nueva clasificación de los sectores productivos, destacando sus relaciones de causalidad (arrastre) y cuantificando mediante varios índices sus poderes de atracción relativos (Morillas, 1995, p. 98).

1 Se considera el trabajo de Morillas (1983) como el estudio inicial de ordenamiento sectorial mediante la teoría de grafos (García y Ramos, 2003: 2).

2 En 2008, el INEGI dio a conocer la Matriz Nacional de Insumo-Producto (MIPMX'03) con información del año 2003. La MIPMX'03 está disponible en:

http://www.inegi.gob.mx/inegi/default. 
El trabajo se divide en siete secciones. Luego de esta introducción se definen algunos conceptos fundamentales de la teoría de grafos, que ofrece la posibilidad de analizar estructuras interdependientes a partir de relaciones binarias cualitativas; en la tercera sección se presenta el grafo asociado al modelo de insumo producto de oferta y demanda; en la cuarta se enuncian los diferentes conceptos relativos a las relaciones de influencia sectoriales; en la quinta se reseña el enfoque tradicional de ordenamiento sectorial y se resaltan sus límites; en la sexta se considera el enfoque de Morillas para la identificación de sectores clave; en la séptima se realiza una nueva clasificación de los sectores a partir de dos indicadores sintéticos de interdependencia, basados en la matriz de caminos asociada al grafo. Por último, se exponen las principales conclusiones.

\section{Grafos}

$\mathrm{Un}_{\text {grafo }}^{3} \mathrm{G}=(\mathrm{X}, \mathrm{U})$ está compuesto por dos conjuntos:

1. Un conjunto $X$, finito, formado por $n$ elementos llamados vértices, nodos o polos.

2. Un conjunto $U$, cuyos elementos son los arcos que pueden definirse entre los elementos de X, que para el caso de los grafos orientados será una parte del producto cartesiano X.X, es decir, $\mathrm{X}^{2}$. La orientación del arco indica el sentido de las relaciones entre los elementos de $\mathrm{X}$.

Si dos vértices, $\mathrm{X}_{\mathrm{i}} \mathrm{y} \mathrm{X}_{\mathrm{j}}$, están relacionados de tal forma que podemos escribir que $\mathrm{xj} \in f(\mathrm{xi})$, siendo $f$ una aplicación de $\mathrm{X}$ en $\mathrm{X}$, existirá una línea orientada que unirá ambos vértices, a la que llamaremos arco. Un arco se puede definir como el par (xi, $\mathrm{xj})$, con $\mathrm{xj} \in f(\mathrm{xi})$, es decir:

$$
\forall \mathrm{xi}, \mathrm{xj} \in \mathrm{X} \text { xi } \mathfrak{R} \mathrm{xj} \Leftrightarrow \mathrm{xj} \in f(\mathrm{xi})
$$

Hay ocasiones en que un vértice puede estar relacionado consigo mismo (propiedad reflexiva). En tal caso, al arco se denomina bucle.

Un grafo valuado es un grafo $\mathrm{G}=(\mathrm{X}, \mathrm{U})$, en el que cada arco está provisto de un valor. Los arcos de un grafo valuado pueden ser valorados de muy distintas maneras. En particular, nos centramos en grafos en los que sus arcos están valorados mediante los coeficientes de un sistema de ecuaciones.

3 Morillas (1983: 160-165; 2004: 85).

Desarrollo 
De lo anterior, un grafo de influencia es una terna $\mathrm{G}=(\mathrm{X}, f, \mathrm{~h})$ donde los vértices $\mathrm{xi} \in \mathrm{X} \forall i=1,2, \ldots, n$ representan las $\mathrm{n}$ variables de un sistema de ecuaciones lineales, $f$ se determina mediante las relaciones entre las variables y h es una aplicación definida como sigue:

$$
\mathrm{h}:\left\{(\mathrm{xi}, \mathrm{xj}) \quad \in \mathrm{X}^{2} / \mathrm{f}(\mathrm{xi})=\mathrm{xj}\right\} \rightarrow \mathfrak{R}
$$

De este modo, $\mathrm{h}:\left\{\left(\mathrm{x}_{\mathrm{i}}, \mathrm{x}_{\mathrm{j}}\right)=\mathrm{h}_{\mathrm{ij}}\right\}$ representa un grafo valuado asociado a un sistema de ecuaciones lineales cuyos arcos están valorados con los parámetros establecidos en ese sistema de ecuaciones.

Esto último permite efectuar una representación de la estructura de un modelo lineal, tanto por su forma más convencional (sistema de ecuaciones), como mediante su correspondiente grafo valuado asociado.

\section{Grafo asociado al modelo de insumo-producto}

El modelo abierto de insumo-producto siempre deberá cumplir la relación contable referente a la igualdad entre la suma de sus ventas y compras totales. En forma matricial, estas ecuaciones de balance pueden especificarse del siguiente modo:

$$
\begin{aligned}
& x=x D+v \\
& x=A x+y
\end{aligned}
$$

donde:

$\mathrm{x}=$ vector $(n x l)$ de producción final.

$\mathrm{Ax}=$ matriz (nxn) de demanda intermedia.

$\mathrm{y}=$ vector $(n x l)$ vector de demanda final.

$\mathrm{xD}=$ matriz $(n x n)$ de distribución del gasto de la producción.

$\mathrm{v}=$ vector $(n x l)$ gastos de insumo primarios.

La solución del conocido modelo de demanda o modelo vertical es:

$$
\mathrm{x}=(\mathrm{I}-\mathrm{A})^{-1} \mathrm{y} \mathrm{x} \geq 0 \quad \forall \mathrm{y} \geq 0
$$

En este caso, la matriz A se refiere a los coeficientes técnicos, y la matriz (I-A) ${ }^{-1}$ es conocida comúnmente como la inversa de Leontief o matriz tecnológica, e indica los insumos directos e indirectos de producción en cada uno de los sectores para compensar el incremento de una unidad en la demanda final. 
La solución del modelo de oferta o modelo horizontal es:

$$
\mathrm{x}^{\mathrm{T}}=\mathrm{v}^{\mathrm{T}}(\mathrm{I}-\mathrm{D})^{-1} \mathrm{x} \geq 0 \quad \forall v \geq 0
$$

En este caso, D es la matriz de coeficientes de distribución de la producción, (I-D) ${ }^{-1}$ es la inversa de la matriz de distribución o de asignación de la producción y $\mathrm{v}^{\mathrm{T}}$ es el vector de insumos primarios transpuesto.

El modelo de insumo producto (dual) puede ser representado mediante un grafo de influencia (Lantner, 1974). La estructura de impulsión de dicho grafo viene dada por las variables exógenas del modelo $\left(\mathrm{y}\right.$ ó $\mathrm{v}^{\mathrm{T}}$ ), que son, necesariamente, vértices fuente del grafo. Las variables endógenas (x) configuran la estructura de respuesta que está compuesta por los restantes vértices del grafo, junto con las relaciones de interdependencia productiva (arcos de grafo) definidas por los coeficientes (A ó D).

En este sentido, el sistema de ecuaciones con base en la matriz D expresa las relaciones de influencia de la oferta (Morillas, 1983: 160-164) ${ }^{4}$. Es decir, cómo el sector productivo $i$ influye al sector productivo $j$ cuando se produce una variación en la producción total del sector $i$ que genera una variación en la producción del sector $j$.

Estas relaciones de influencia pueden definirse ya sea en términos absolutos o relativos a la producción total de cada sector, y en términos directos o globales según se tengan en cuenta los flujos de compra y venta directas o globales. No obstante, en la presente investigación sólo nos centraremos en términos relativos, puesto que las variaciones relativas suelen ser más útiles que las absolutas ${ }^{5}$.

4 El paso de un tipo de influencia a otra es inmediato. Si se desea estudiar la influencia por el lado de la demanda, bastará con invertir la orientación de los arcos (principio de dualidad direccional). Sin embargo, en los resultados que se presentan, se trabajará con los elementos de la matriz D, que entre otras ventajas pondera los coeficientes de la matriz A con las producciones de los respectivos sectores, resultando así en una definición de la influencia basada en la posición y el peso relativo de cada sector dentro de la estructura de intercambios más que en el simple coeficiente técnico $\left(\mathrm{d}_{\mathrm{ij}}=\mathrm{a}_{\mathrm{ij}}\left(\mathrm{x}_{\mathrm{j}} / \mathrm{x}_{\mathrm{i}}\right)\right)$.

5 De hecho Lantner considerado como una autoridad intelectual en este tema opina que el grafo de influencia en sentido estricto es el de la influencia relativa (Lantner, 1974: 68). 


\section{Relaciones de influencia en el grafo asociado}

El concepto de influencia relativa del sector $i$ sobre el sector $j$, denotada por $\mathrm{I}^{\mathrm{R}}(\mathrm{i}) \rightarrow \mathrm{j}$, nos indica el incremento relativo en la producción del sector j cuando se produce un aumento relativo de la producción del sector $i$, es decir,

$$
\mathrm{I}_{(\mathrm{i}) \rightarrow \mathrm{j}}^{\mathrm{R}}=\frac{\Delta \mathrm{xj} / \mathrm{xi}}{\Delta \mathrm{xi} / \mathrm{xi}}
$$

y establecemos que $i \mathrm{I}^{\mathrm{R}} j$, si $\mathrm{d}_{\mathrm{ji}} \neq 0$, donde $\mathrm{d}_{\mathrm{ij}}$ representa el $i, j$-ésimo coeficiente de la matriz de distribución (D); es decir, si el sector $i$ compra directamente a la rama $j$.

En particular, la expresión de la influencia directa relativa para el caso de dos sectores $i, j$ será,

$$
\left(\frac{\Delta \mathrm{xj}}{\mathrm{xj}}\right)=d_{j i}\left(\frac{\Delta \mathrm{xi}}{\mathrm{xi}}\right)
$$

y establecemos que i ID $\mathrm{j}$, si $\operatorname{dji}^{1} 0$, donde el elemento $\mathrm{d}_{\mathrm{ij}}$ puede ser considerado como una elasticidad ${ }^{6}$.

En tanto, la influencia global relativa (Morillas, 2004: 88) será,

$$
\hat{\chi}-1 \Delta x=(\mathrm{I}-\mathrm{D})^{-1} \hat{\mathrm{e}} \hat{\mathrm{y}}^{-1} \Delta y
$$

donde:

$\chi-1=$ vector diagonal de productos totales por sector.

$\hat{e}=x^{-1} y=$ coeficiente de demanda final (tasa de exportación fuera de la estructura del grafo).

$(\mathrm{I}-\mathrm{D})^{-1}=$ inversa de la matriz de distribución.

Más aún, la influencia global en términos relativos del sector i sobre el sector j se puede obtener por el desarrollo de inversión de matrices conocido como método de expansión de potencias.

$$
\Pi=(\mathrm{I}-\mathrm{D})^{-1}=\left(\mathrm{I}+\mathrm{D}+\mathrm{D}^{2}+\mathrm{D}^{3}+\ldots+\mathrm{D}^{\mathrm{n}}\right)
$$

Así, si se tienen en cuenta las transacciones directas e indirectas, podemos afirmar que el sector $i$ influye globalmente en términos relativos al sector $j, i \mathrm{I}^{\mathrm{G}} j$, si $\pi_{\mathrm{ij}} \neq 0$; siendo $\pi_{\mathrm{ij}}$ el $i, j$-ésimo coeficiente de la matriz denotada por П. (García y Amo, 2000: 4).

6 La expresión que cuantifica la influencia directa en términos absolutos del sector i sobre el sector $\mathrm{j}$, se puede escribir como:

$$
\Delta \mathrm{x}_{\mathrm{j}}=d_{j i} \Delta \mathrm{x}_{\mathrm{i}}
$$

siendo $d_{i j}$ un elemento genérico de la matriz $(\mathrm{I}-\mathrm{D})^{-1}$. 


\section{El método clásico}

El análisis estructural basado en los modelos insumo-producto tiene su origen en los trabajos pioneros de Hirschman (1958), Chenery y Watanabe (1958), Rasmussen (1956) y Ghosh (1958). Posteriormente, el esfuerzo fue continuado con otras metodologías como la extracción hipotética, iniciada por Strassert (1968) y seguida por otros autores como Cella (1984), Dietzenbacher y Van Der Linden (1997), entre en otros?

El método clásico del análisis estructural desarrollado por Chenery y Watanabe (1958), en líneas generales, se centra en la cuantificación de los encadenamientos hacia atrás (BL, por sus siglas en inglés) y hacia adelante (FL) y a partir de ellos se establece una clasificación cuatripartita de los sectores según sus encadenamientos hacia atrás y hacia adelante.

Cuadro 1.

Tipificación de sectores según sus encadenamientos

\begin{tabular}{|c|c|c|}
\hline & BL $<$ Promedio & BL $>$ Promedio \\
\hline$F L>$ Promedio & Base o Estratégico & Clave \\
\hline$F L<$ Promedio & Independientes & Impulsores \\
\hline
\end{tabular}

Fuente: Elaboración Propia.

Conceptualmente, los sectores base presentan unos encadenamientos hacia atrás menores que el promedio y hacia delante por encima de la media; éstos generan una escasa demanda, pero mucha oferta. Los sectores impulsores de la economía demandan insumos de otros sectores intermedios, consecuentemente, generan un estímulo a la producción de bienes intermedios. Los sectores independientes o aislados en general son poco atractivos, ya que provocan un menor impacto en la economía, pues su desarrollo no afecta significativamente a los sectores que demandan sus productos, ni a los que los utilizan como productos intermedios. En tanto, los sectores clave presentan unos encadenamientos hacia atrás y hacia adelante mayores al promedio; son importantes por la demanda que producen y la oferta que generan en el resto de los sectores.

Chenery y Watanabe (1958) utilizan el modelo de demanda o modelo vertical de insumo producto para proponer unas medidas a partir de las cuales es posible determinar los encadenamientos directos hacia atrás $\left(\mathrm{BL}^{\mathrm{Ch}-\mathrm{w}}\right)$ y hacia adelante $\left(\mathrm{FL}^{\mathrm{Ch}-\mathrm{w}}\right)$ entre los distintos sectores productivos de una economía. Estas medidas se pueden

7 El autor propone cuantificar el efecto que se produciría en una economía si se extrajera hipotéticamente de ella un determinado sector, de esta manera realiza lo que se denomina Análisis Estructural de Extracción de Sectores. (Strassert, 1958). 
expresar en términos relativos para facilitar la comparación entre distintas situaciones, es decir:

$$
\mathrm{BL}^{\mathrm{Ch}-\mathrm{w}}=\frac{i^{\prime} A}{i^{\prime} A i / n} \quad \mathrm{FL}^{\mathrm{Ch}-\mathrm{w}}=\frac{A i}{i^{\prime} A i / n}
$$

donde:

i' = vector fila con valores iguales a la unidad.

$\mathrm{A}=$ matriz de coeficientes técnicos.

$\mathrm{i}=$ vector columna con valores iguales a uno.

Posteriormente, Rasmussen (1956) sugiere dos medidas basadas en la matriz inversa de Leontief para cuantificar los efectos directos e indirectos hacia atrás $\left(\mathrm{BL}^{\mathrm{R}}\right)$ y adelante $\left(\mathrm{FL}^{\mathrm{R}}\right)$ que puede experimentar un sector. Las medidas normalizadas se obtienen a partir de las expresiones siguientes:

$$
\mathrm{BL}^{\mathrm{R}}=\frac{n i^{\prime}(I-A)^{-1}}{i^{\prime}(I-A)^{-1} i} \quad \mathrm{FL}^{\mathrm{R}}=\frac{n(I-A)^{-1} i}{i^{\prime}(I-A)^{-1} i}
$$

donde:

$(\mathrm{I}-\mathrm{A})^{-1}=$ matriz inversa de Leontief.

La primera medida se denomina poder de dispersión; la segunda, sensibilidad de dispersión.

Por último, Ghosh (1958), empleando el modelo de oferta o modelo horizontal de insumo producto, propone unas medidas de ordenamiento sectorial a partir de los encadenamientos hacia delante ${ }^{8}$. Las medidas de los encadenamientos hacia adelante, directos y globales, son:

donde:

$$
F L_{G}^{C h-W}=D i \quad F L_{G}^{R}=\frac{n(I-D)^{-1} i}{i^{\prime}(I-D)^{-1} i}
$$

$\mathrm{D}=$ matriz de distribución de producción

$(\mathrm{I}-\mathrm{D})^{-1}=$ inversa de la matriz de distribución.

8 Respecto a estas medidas algunos autores señalan que es más adecuado adoptar el modelo de oferta y redefinir, a partir de éste, los encadenamientos hacia adelante, ya que éstos se refieren a un crecimiento de la oferta generado por un aumento en la demanda, esto es, al crecimiento de una unidad de las ventas finales y, tal como se han presentado hasta ahora, cuantifican bien un crecimiento simultáneo de una unidad en el producto de cada sector (Chenery y Watanabe), bien el aumento de una unidad en la demanda final (Rasmussen). (Soza, 2004: 38). 
Sin embargo, a pesar de la sencillez y claridad en la interpretación, estos métodos tradicionales son susceptibles de mejoramiento, en la medida en que el análisis de los indicadores originales utiliza exclusivamente el peso económico de los encadenamientos directos, que si bien facilitan la comparación entre sectores porque "aíslan" la fuente de las diferencias, no consideran el grado de concentración de un determinado sector. También porque no basta sólo con incorporar los encadenamientos globales (directos e indirectos), pues aunque es correcto que el uso de la matriz inversa suministra información muy útil y más genuina que la matriz de coeficientes técnicos, no nos dice cómo unos sectores pueden estar más interconectados que otros ${ }^{9}$. Finalmente, estas medidas no cuantifican la relevancia concreta de las relaciones productivas de cada sector respecto a los demás y omiten la relación jerárquica entre ellos en la transmisión de los impulsos dentro de la estructura de intercambios (Morillas, 1995: 94) ${ }^{10}$.

\section{El método de grafos}

El análisis estructural de insumo-producto moderno tiene su origen en los trabajos de Ponsard (1972) y Lantner (1974) y ha sido continuado por Morillas (1983, 1995, 2004), García y Ramos (2003, 2006) y García, Morillas y Ramos (2005), entre otros.

Morillas (1995) propone cuantificar cómo se relacionan productivamente los diferentes sectores, a partir de unos índices que determinan el poder de atracción relativa entre los distintos sectores de la economía, teniendo como base la matriz de coeficientes de distribución de la producción nacional (D) (efectos directos) y la inversa de distribución (I- D) $)^{-1}$ (efectos globales).

Para ello, el autor parte del método de expansión de potencias, lo cual permite separar el efecto directo y los efectos indirectos. El efecto global se descompone sumando las rondas del proceso convergente ${ }^{11}$ :

$$
\Pi=(\mathrm{I}-\mathrm{D})^{-1}=\left(\mathrm{I}+\mathrm{D}+\mathrm{D}^{2}+\mathrm{D}^{3}+\ldots+\mathrm{D}^{\mathrm{n}}\right)
$$

9 De hecho, los índices de Rasmussen sólo nos informan sobre los encadenamientos globales de términos tecnológicos. Hazari (1970) considera que tenemos que ponderar los encadenamientos en función de su contribución a la demanda final. (Fuentes y Sastré, 2001: 154; Aroche-Reyes, 2005: 33).

10 Es importante determinar no sólo el peso que tiene cada sector en el sistema económico, sino también cómo se relacionan los diferentes sectores en el entramado económico. (García y Ramos, 2003: 2).

11 Para que se realice la convergencia, se deben cumplir las condiciones Hawkins-Simon (que los menores principales de la matriz (I-D) sean positivos). (Kozikowski, 1984: 130). 
donde:

(I + D) es la suma del efecto inicial (I) y el efecto directo (D).

$\left(D^{2}+D^{3}+\ldots+D^{n}\right)$ es la suma de efectos indirectos.

De donde cada elemento $\mathrm{d}_{\mathrm{ij}}$ de la matriz D representará las ventas directas en términos relativos del sector $i$ al sector $j$ en relación con el producto total del sector $i$ (por renglón). Análogamente, dji representa las compras directas del sector $j$ del bien producido por el sector i en proporción a la producción total de este sector (por columnas) ${ }^{12}$.

Por tanto, los elementos genéricos representan un índice de dependencia del sector $i$ respecto de las compras de su producción efectuadas por el sector $j$ (filas) o del sector $j$ respecto de la producción ofrecida por el sector $i$ (columnas).

Ahora, como el atributo principal que nos interesa es la interdependencia de las relaciones entre el conjunto de sectores, podemos definir a la matriz adyacente asociada al grafo A(D) como:

$$
\mathrm{A}(\mathrm{D})= \begin{cases}\mathrm{d}_{\mathrm{ij}}=1 & \text { si existe la relación entre el sector } i \text { y } j \\ \mathrm{~d}_{\mathrm{ij}}=0 & \text { si no existe relación entre el sector } i \text { y } j\end{cases}
$$

También, podemos definir la matriz de caminos, R(D), como una matriz cuadrada de orden $n$ cuyos elementos $\mathrm{d}_{\mathrm{ij}}$ pueden tomar los valores 1 y 0 .

$$
\mathrm{R}(\mathrm{D})= \begin{cases}\mathrm{d}_{\mathrm{ij}}=1 & \text { si existe la relación entre el sector } i \text { y } j \\ \mathrm{~d}_{\mathrm{ij}}=0 & \text { si no existe camino entre el sector } i \text { y } j\end{cases}
$$

De ahí que las relaciones indirectas que tienen en cuenta la interdependencia global de todos los sectores en el grafo vienen dadas por las potencias sucesivas de la matriz de caminos: $\mathrm{D}^{2}$ para caminos de longitud $2, \mathrm{D}^{3}$ para caminos de longitud $3, \ldots$, $\mathrm{D}^{\mathrm{n}}$ para caminos de longitud $n$.

De lo anterior se desprende, por ejemplo, que el elemento genérico de $\mathrm{D}^{2}, \mathrm{~d}_{\mathrm{ij}}{ }^{(2)}=\sum_{i k} d_{i k} d_{k j}$ nos muestra las ventas, en términos relativos, del sector $i$ al sector $j$, a través dé los $n$ sectores de la economía, para aquellos caminos de longitud 2. De esta manera, las relaciones directas e indirectas entre sectores, para caminos de longitud $n$, vendrán dadas por la matriz de medidas de atracción $\mathrm{M}$, tal que ${ }^{13}$ :

$$
\mathrm{M}=\mathrm{D}+\mathrm{D}^{2}+\mathrm{D}^{3}+\ldots+\mathrm{D}^{\mathrm{n}}=\sum_{k=1} \mathrm{D}^{k}
$$

12 El elemento $i$-ésimo de la matriz D se define como:

$$
\mathrm{d}_{i j}=\frac{x_{i j}}{x_{i}}
$$

13 Al venir dada la influencia directa a lo largo de un camino por el producto de las valoraciones de los arcos, los elementos mij de la matriz M serán un índice de interacción global existente entre los sectores i y j. (Morillas, 1995: 96). 
Esta expresión se puede escribir como:

$$
\mathrm{M}=\mathrm{D}\left(\mathrm{I}+\mathrm{D}+\mathrm{D}^{2}+\ldots+\mathrm{D}^{\mathrm{n}-1}\right)=\mathrm{D}(\mathrm{I}-\mathrm{D})^{-1}
$$

En términos conceptuales, debido a que D es una matriz de medidas de atracción directa, la matriz M nos indica los efectos directos e indirectos de tal atracción y sus elementos $\mathrm{m}_{\mathrm{ij}}$ muestran el total de efectos entre $i \mathrm{y} j$, incluyendo todas las posibles relaciones intermedias con el resto de los sectores.

Los vectores que permiten obtener un ordenamiento sectorial tienen que ver con la suma por columnas de la matriz $\left(\mathrm{c}=\mathrm{M}_{\mathrm{i}}^{\mathrm{T}}\right)$, que denota un índice sectorial de atracción global por compras y análogamente con la suma por filas $\left(\mathrm{v}=\mathrm{M}_{\mathrm{i}}\right)$, que constituye un índice sectorial de atracción global por ventas. Sin embargo, para evitar la existencia de circuitos en el grafo se imponen las siguientes condiciones:

1. Un sector será núcleo si sus flujos de primer y segundo orden (primer y segundo máximo) están dirigidos hacia sectores con menor atracción global.

2. Un sector $j$ estará subordinado al sector $i$ si el flujo máximo o el flujo secundario de $j$ está dirigido hacia $i$, teniendo este sector $i$ una atracción global superior a la de $j$.

3. Como corolario de las anteriores condiciones, un sector no puede ser subordinado a otro de menor atracción global.

Para la obtención del ordenamiento sectorial en forma de grafo de los sectores vendedores tenemos que realizar los siguientes pasos:

1. Ordenar los sectores según sus índices de atracción global por compras $\left(\mathrm{c}_{\mathrm{j}}\right)$

2. Identificar los flujos primario $\left(\mathrm{m}_{\mathrm{kjm}}\right)$ y secundario $\left(\mathrm{m}_{\mathrm{ljs}}\right)$ de las ventas de cada sector $i$.

3. En caso de que los índices de atracción global de los sectores jm y js sean menores que los del sector $i$, este último se denota como núcleo. Es decir, que será núcleo si y sólo si:

$$
\left.\begin{array}{l}
\mathbf{c}_{i}>\mathbf{c}_{j m} \\
\mathbf{c}_{i}>\mathbf{c}_{j s}
\end{array}\right\} \Rightarrow i \text { es un verdadero núcleo }
$$

4. $\mathrm{Si}_{\mathrm{kj}}=\mathrm{m}_{\mathrm{ki}}$ con $\mathrm{c}_{\mathrm{i}}>\mathrm{c}_{\mathrm{k}}$, entonces el sector $k$ estará subordinado al $i$ por sus ventas.

5. Si existe un sector $k$ cuyo flujo secundario $\left(\mathrm{m}_{\mathrm{kjs}} \geq{ }_{\mathrm{min}} \mathrm{m}_{\mathrm{ijm}}\right.$ ), y siempre que se respeten los vínculos de subordinación, entonces $k$ también está subordinado a $j$ por sus ventas. 


\section{Análisis de la economía mexicana}

Como paso previo a la jerarquización sectorial de acuerdo con la influencia relativa concreta de cada sector respecto a los demás en el sistema productivo, debemos aclarar las bases de partida para poder valorar los resultados en sus justos términos.

- La Matriz de Insumo Producto Nacional está referida al año 2003. Como todos sabemos, en 2008, el INEGI dio a conocer la MIPMX'03 con información de 2003 en dos presentaciones: una conformada por 20 sectores y la otra por 79 subsectores de actividad. La MIPMX'03 se ha construido de acuerdo con la normativa del Sistema de Clasificación Industrial para América del Norte (SCIAN) y experimenta un cambio metodológico con respecto a publicaciones anteriores, ya que se ha modificado la clasificación de actividades, al sustituir el Sistema de Clasificación Nacional Mexicano de 1993 (SCNM-93) ${ }^{14}$.

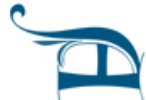

- El estudio empírico se basa en la presentación de la MIPMX'03 a 20 sectores. La matriz cubre más del $95 \%$ de la población y del valor bruto de la producción nacional, por lo que es representativa para el análisis estructural.

- La tipificación sectorial elegida para la presentación de los resultados empíricos nacionales, relativos al análisis clásico, fue la propuesta por Rasmussen (1956), ya que estos índices perfeccionan los de Chenery y Watanabe (1958), al incorporar los efectos de encadenamiento global que se producen entre los sectores tanto por el lado de la oferta como del de la demanda; asimismo, incluyen el efecto de dispersión sectorial e incorporan ponderaciones en los encadenamientos sectoriales de acuerdo con su importancia relativa en la demanda final.

En la gráfica 1 se muestran los resultados de la tipificación sectorial referida a los encadenamientos globales sectoriales con base en los criterios de Rasmussen: cinco de ellos se clasifican como actividades base o estratégicas; siete actividades son independientes; cuatro se encuentran clasificadas como impulsoras y, finalmente, sólo cuatro actividades forman parte del grupo de sectores clave.

En el grupo de sectores clasificados como base o estratégicos -llamados así por la importancia de sus ventas intersectoriales-- hay que resaltar la presencia de actividades como comercio (6), transporte (7), servicios inmobiliarios (11), servicios profesionales (12) y servicios de apoyo a negocios (14).

14 Fruto de dicho cambio es la variación experimentada en la composición y número de sus sectores, así pues, mientras la MIPMX' 80 aparece clasificada en 72 ramas, la MIPMX'03 se agrega en 79 ramas de actividad. 
Gráfica 1.

Clasificación de sectores según

sus encadenamientos Rasmussen

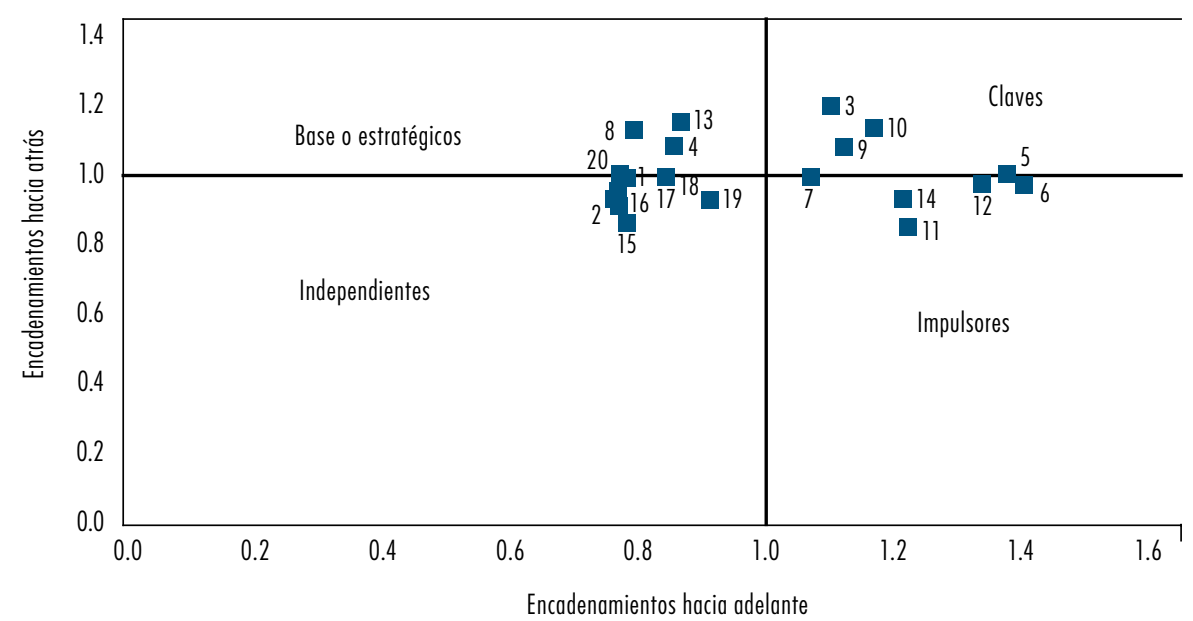

El grupo de sectores clave - caracterizados por ser fuertes demandantes de insumos intersectoriales y oferentes de productos intermedios, es decir, paso obligado de los flujos sectoriales de la economía - está compuesto por las actividades de electricidad, gas y agua (3), industrias manufactureras (5), información en medios masivos (9) y servicios financieros y de seguros (10).

El grupo de los sectores impulsores — denominados así por la relevancia de sus compras intersectoriales - incluye la construcción (4), correos y almacenamiento (8), dirección de corporativos (13) y actividades de gobierno (20).

Dentro del grupo de sectores independientes - caracterizados por sus débiles articulaciones tanto de compras como ventas - se encuentra la agricultura (1), minería (2), servicios de educación (15), servicios de salud (16), servicios de esparcimiento (17), hoteles, alimentos y bebidas (18), y otros servicios (19).

Establecido lo anterior, se analizarán los resultados de la aplicación del método de grafos a la economía nacional. En los cuadros 2 y 3 se presentan las jerarquías sectoriales por ventas y compras según los índices sintéticos de atracción.

En el grupo de sectores tipificados como de alto poder de atracción por sus ventas al resto de la economía, hay que destacar la presencia de actividades como dirección de corporativos (13), servicios de apoyo a negocios (14), servicios profesionales (12), electricidad, gas y agua (3) y servicios financieros (10), sectores conocidos por ser proveedores, directos o indirectos, de los demás sectores productivos, de tal forma que su producción es absolutamente necesaria para el funcionamiento económico nacional. 
Cuadro 2

Ordenamiento sectorial

según la atracción global de sus ventas

\begin{tabular}{|c|c|c|c|}
\hline Rango & Sectores & Índice Vii & $\begin{array}{l}\text { Índice relativo de } \\
\text { concentración sectorial }\end{array}$ \\
\hline 1 & s13. Dirección de corporativos & 1.5893 & 72 \\
\hline 2 & s74. Servicios de apoyo a negocios & 1.3011 & 22 \\
\hline 3 & s12. Servicios profesionales & 1.0215 & 21 \\
\hline 4 & s3. Electricidad, agua y gas & 0.9759 & 27 \\
\hline 5 & s10. Servicios financieros & 0.8804 & 16 \\
\hline 6 & s8. Correos y almacenamiento & 0.8201 & 17 \\
\hline 7 & s2. Minería & 0.7616 & 100 \\
\hline 8 & sl. Agricultura, ganadería, pesca, y caza & 0.7505 & 84 \\
\hline 9 & s9. Comunicación/información en medios & 0.6614 & 8 \\
\hline 10 & s6. Comercio & 0.5174 & 28 \\
\hline 11 & s5. Industrias manufactureras & 0.3979 & 19 \\
\hline 12 & s7. Transportes & 0.3854 & 17 \\
\hline 13 & s19. Otros servicios & 0.3760 & 8 \\
\hline 14 & s11. Servicios inmobiliarios & 0.3533 & 6 \\
\hline 15 & s18. Hoteles, alimentos y bebidas & 0.1802 & 5 \\
\hline 16 & s4. Construcción & 0.0978 & 10 \\
\hline 17 & s17 Servicios de esparcimiento & 0.0430 & 4 \\
\hline 18 & s20. Actividades del gobierno & 0.0221 & 1 \\
\hline 19 & s15. Servicios educativos & 0.0175 & 1 \\
\hline \multirow[t]{4}{*}{20} & s16. Servicios de salud & 0.0000 & 0 \\
\hline & Promedio & 0.5576 & \\
\hline & Varianza & 0.2058 & \\
\hline & Coeficiente de variación & 0.3690 & \\
\hline
\end{tabular}

Fuente: Cálculos Propios con base en la MIPMX'03.

* / Coeficiente de variación mayor $=100$. El coeficiente de variación por filas mide la dispersión de las ventas de un sector entre los restantes en unidades de la media de ventas en ese sector. Se expresan los coeficientes de variación en números índices con la finalidad de dar una idea precisa de la mayor o menor de los efectos de un sector en el conjunto de la economía: 100 es el índice que nos dice cuál es el sector cuyos efectos están más concentrados en determinados sectores.

Conviene aclarar que, de acuerdo con el índice relativo de concentración sectorial, las actividades de agricultura, ganadería, caza y pesca (1), minería (2) y dirección de corporativos (13) tienen una menor difusión de sus efectos por sus ventas en la medida que están muy concentradas en determinados sectores.

La jerarquía del grupo de sectores según el índice de atracción global por las compras está compuesta por: actividades manufactureras (5), comercio (6), construcción (4), transportes (7), información en medios (9) y servicios financieros (10). El orde- 
namiento se caracteriza por una mayor polarización, como índica su alto coeficiente de variación. Estos sectores son, consecuentemente, los que tienen mayor capacidad global para transmitir los impulsos de su actividad productiva mediante las compras efectuadas al resto de la economía. En este grupo es importante observar que la manufactura presenta un alto grado de concentración de sus efectos.

Cuadro 3.

Ordenamiento sectorial

Según la atracción global de sus compras

\begin{tabular}{|c|c|c|c|}
\hline Rango & Sectores & Índice Cii & $\begin{array}{l}\text { Índice relativo de } \\
\text { concentración sectorial }\end{array}$ \\
\hline 1 & s5. Industrias manufactureras & 4.1963 & 63 \\
\hline 2 & s6. Comercio & 1.1275 & 28 \\
\hline 3 & s4. Construcción & 0.7894 & 6 \\
\hline 4 & s9. Comunicación/información en medios & 0.7654 & 100 \\
\hline 5 & s7. Transportes & 0.6579 & 9 \\
\hline 6 & s10. Servicios financieros & 0.5788 & 25 \\
\hline 7 & s20. Actividades del gobierno & 0.4985 & 13 \\
\hline 8 & s2. Minería & 0.3684 & 17 \\
\hline 9 & s3. Electricidad, agua y gas & 0.3627 & 24 \\
\hline 10 & sl. Agricultura, ganadería, pesca, y caza & 0.3148 & 11 \\
\hline 11 & s12. Servicios profesionales & 0.2770 & 6 \\
\hline 12 & s1l. Servicios inmobiliarios & 0.2610 & 9 \\
\hline 13 & s18. Hoteles, alimentos y bebidas & 0.2334 & 6 \\
\hline 14 & s16. Servicios de salud & 0.1603 & 4 \\
\hline 15 & s15. Servicios educativos & 0.1539 & 4 \\
\hline 16 & s19. Otros servicios & 0.1417 & 2 \\
\hline 17 & s74. Servicios a los negocios & 0.1244 & 3 \\
\hline 18 & s13. Dirección de corporativos & 0.0795 & 3 \\
\hline 19 & s17. Servicios de esparcimiento & 0.0372 & 1 \\
\hline \multirow[t]{3}{*}{20} & s8. Correos y almacenamiento & 0.0243 & 0 \\
\hline & Promedio & 0.5576 & \\
\hline & Varianza & 0.7773 & \\
\hline
\end{tabular}

Fuente: Cálculos propios con base en la MIPMX’03.

*_/ Coeficiente de variación mayor $=100$.

Como complemento a lo anterior, se muestran los cálculos concernientes a los flujos primario $\left(\mathrm{m}_{\mathrm{kjm}}\right)$ y secundario $\left(\mathrm{m}_{\mathrm{ljs}}\right)$ de las ventas y compras de cada sector, para detectar la subordinación sectorial. En la figura 1 se presentan los resultados del grafo. 
Figura 1.

Grafo de las relaciones más importantes de la economía mexicana $(5 \%)$

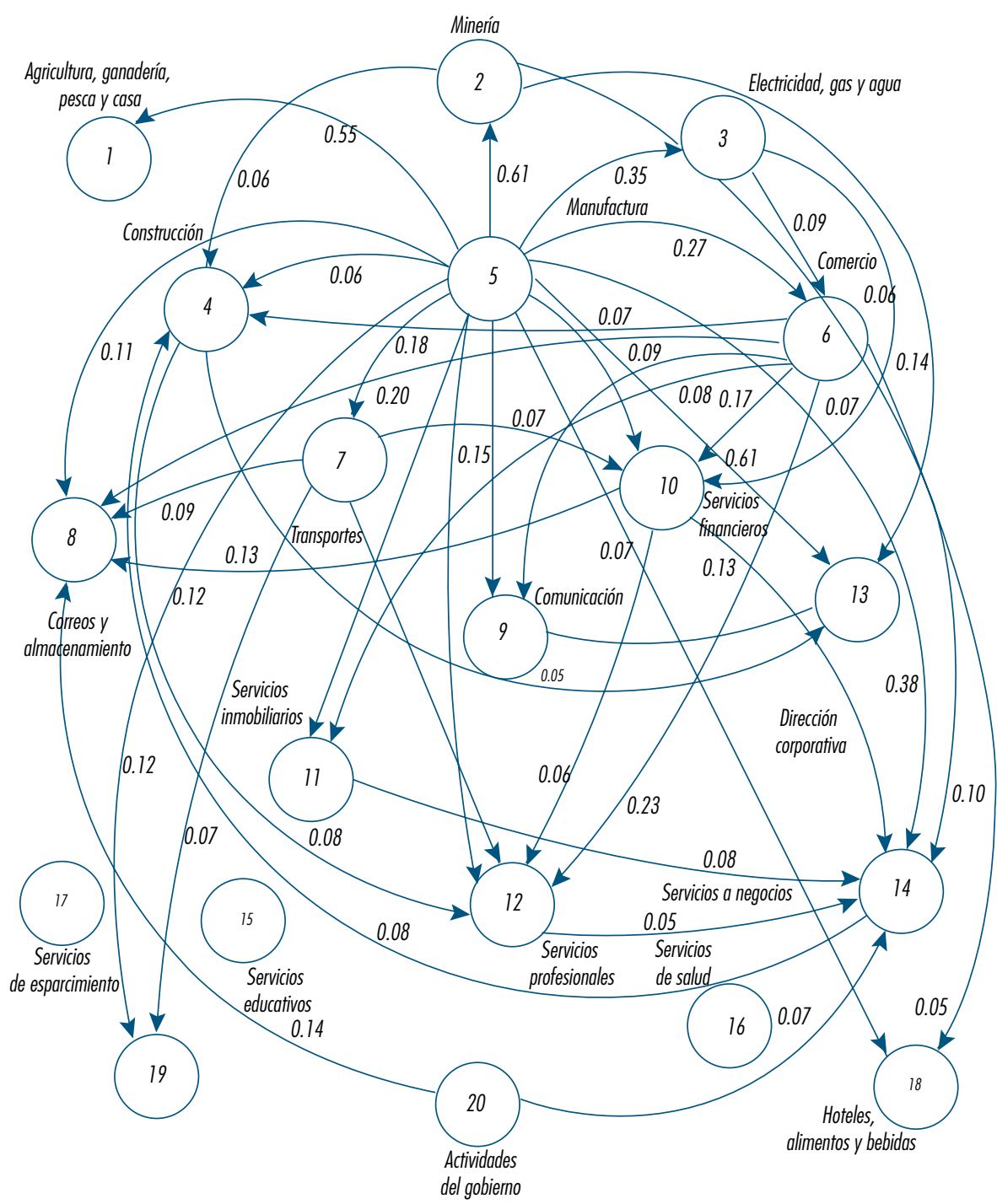

La figura 2 exhibe el grafo parcial de los sectores vendedores. En él los sectores opuestos serán sectores independientes por sus ventas y de gran poder de atracción por sus compras. Los polos de atracción de primer orden -independientes por sus ventas - son manufactura (con 14 relaciones de compra y una de venta), comercio 
(con ocho relaciones de compra y una de venta) y construcción (con cinco relaciones de compra y una de venta), y de segundo orden: transporte (con cuatro relaciones de compra y una de ventas) ${ }^{15}$.

Figura 2.

Grafo de sectores vendedores y sus núcleos de atracción

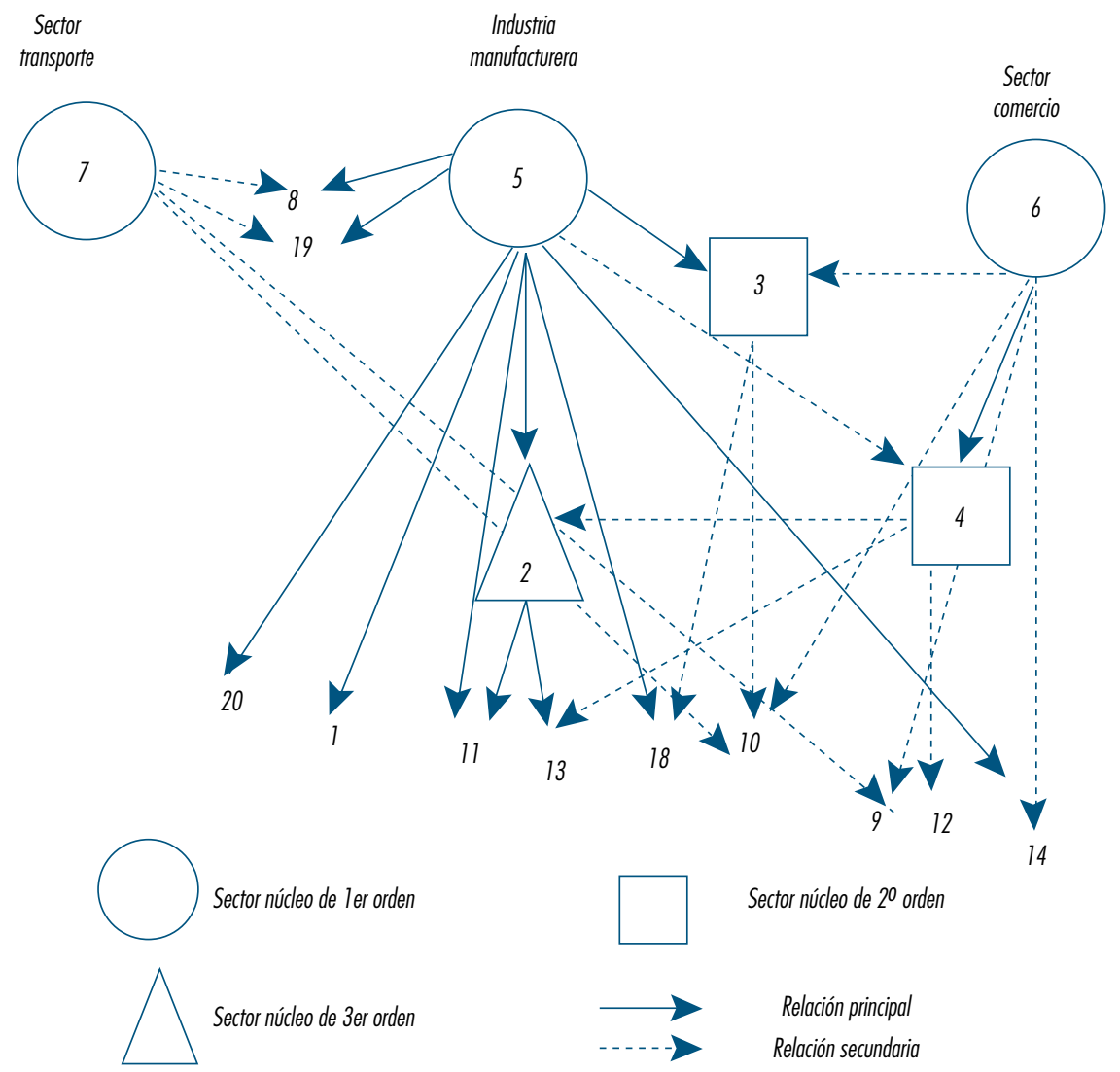

15 Es importante aclarar que los resultados obtenidos están condicionados por el nivel de agregación, división sectorial e importancia de las relaciones de los coeficientes. Existe una amplia tradición que sólo por encima de un valor igual o superior al 0.05 puede considerarse que los coeficientes son representativos de relaciones medias o fuertes en la estructura, catalogando al resto como débiles o, incluso, manifiestamente despreciables (Morillas, 2004: 106)

\section{Desarrollo}


Estos sectores se caracterizan porque sus producciones se dirigen esencialmente hacia la demanda final (inversión, consumo y formación en bruto de capital) y porque compran, directa e indirectamente, de tal forma que su actividad transmite impulsos muy importantes al resto de la economía.

En el grafo se tiene como camino más importante o de primer orden, por el número de relaciones de ventas, al sector de dirección de corporativos (13), servicios de apoyo a negocios (14), servicios profesionales (12), electricidad, gas y agua (3) y servicios financieros y seguros (10). Y de segundo orden, el sector de correos y almacenamiento (8).

Los tres caminos principales de ventas por los que transitan los intercambios sectoriales:

1. Aquel cuyo sector núcleo dominante es el sector manufacturas y los subordinados minería (2), electricidad, gas y agua (3), construcción (4), comunicación e información a medios (9), servicios financieros y de seguros (10), servicios inmobiliarios (11), servicios profesionales (12), dirección de corporativos (13), servicios de apoyo a los negocios (14), hoteles, alimentos y bebidas (18), otros servicios (19) y actividades de gobierno (20).

2. El camino formado por el comercio (6) como núcleo comprador, por el sector construcción (4) que es por donde transita el intercambio sectorial y el sector electricidad, agua y gas (3), servicios financieros y de seguros (10), hoteles, alimentos y bebidas (18), comunicación e información a medios (9), servicios profesionales (12) y servicios de apoyos a los negocios (14) como vendedores.

3. El camino formado por el sector de transporte (7) como sector polarizante de los sectores correos y almacenamiento (8), servicios financieros y de seguros (10), servicios profesionales (12) y otros servicios (19).

En la figura 3 se muestra el grafo de los sectores compradores. En él se exhiben los sectores independientes por sus compras y de gran poder de atracción por sus ventas. En otras palabras, para realizar su función productiva no necesitan otros sectores productivos y, por tanto, sus componentes de costes más importantes serán el valor agregado o las importaciones.

Los núcleos dominantes por sus ventas son servicios profesionales (12), dirección de corporativos (13) y servicios de apoyo a negocios (14). 
El grafo de los sectores compradores destaca que son cuatro los caminos fundamentales por los que transitan los intercambios sectoriales:

1. El que tiene como camino más importante por el número e intensidad de relaciones es el sector de servicios de apoyo a negocios (14), que es un núcleo y que a su vez polariza a actividades como servicios inmobiliarios (11) y manufactura (5); cuyo núcleo de segundo orden son los servicios financieros y de seguros (10), que a su vez es polo de dos sectores de gran importancia como transporte (7) y correos y almacenamiento (8).

Figura 3.

Grafo de sectores compradores y sus núcleos de atracción

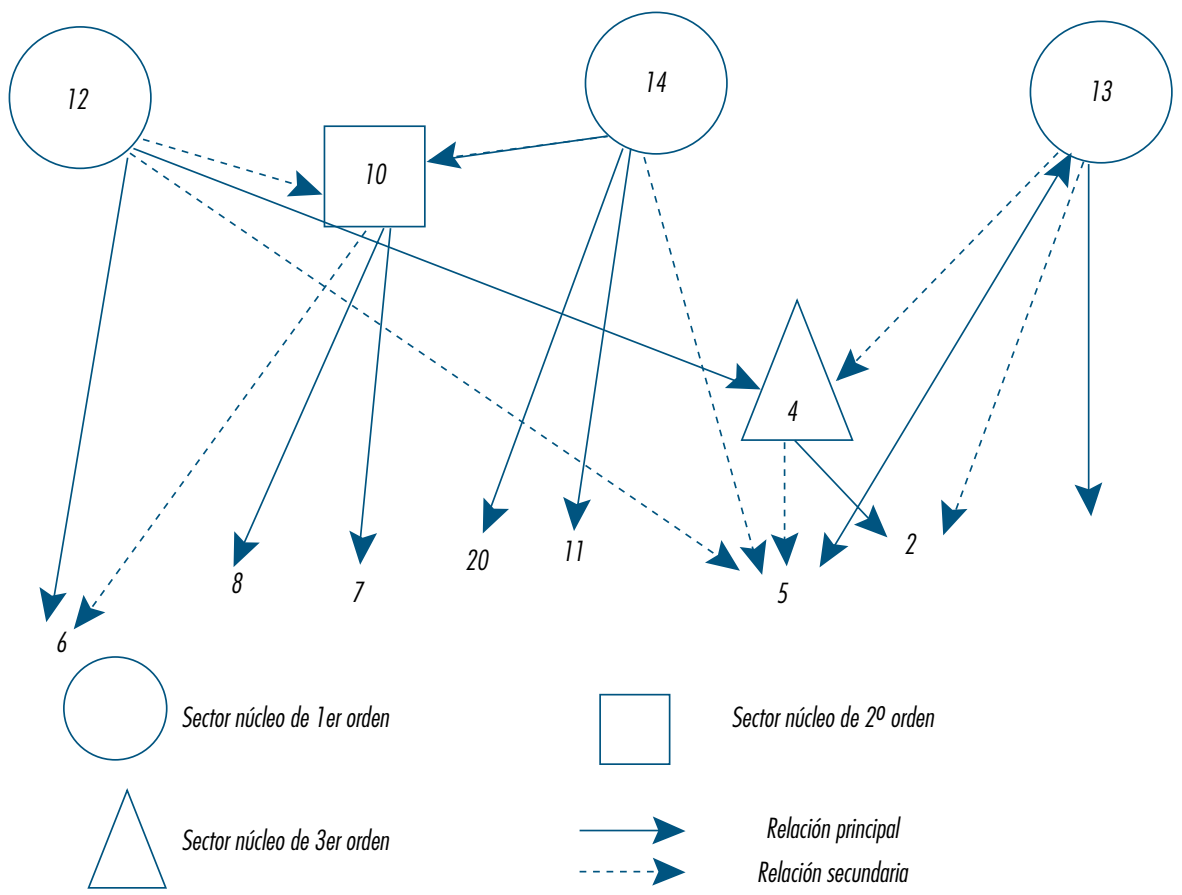

2. El que tiene como base al sector servicios profesionales, pues sus clientes lo utilizan como insumo primario en sus estructuras de coste; es un polo de atracción de orden inferior del sector comercio (6), industria de manufactura (5), construcción (4) y servicios financieros y de negocios (10).

3. El camino formado por el sector de dirección de corporativos (13) como núcleo vendedor, el de construcción (4) - por donde transita el intercambioy el sector minería (2) e industria de manufactura (5) como compradores; 
donde el sector dirección de corporativos (13) a su vez polariza al sector de comunicación e información a medios (9).

4. Los caminos comunes para los dos núcleos principales (servicios profesionales (12) y servicios de apoyo a negocios (14) que se dirigen al sector servicios financieros y de seguros (10). Así como los núcleos principales que acuden al sector de dirección de corporativos (13).

Sintetizando, el grupo formado por su importancia relativa en cuanto a las relaciones de ventas está compuesto por tres núcleos básicos: servicios profesionales (12), dirección de corporativos (13) y servicios de apoyo a negocios (14), en tanto que el grupo de sectores importantes por sus compras está conformado por dos: industrias manufactureras (5) y comercio y transportes. Así, el circuito económico más importante es el conformado por comercio (6) y manufactura (5) con sus servicios básicos.

Finalmente, si incorporamos el factor de la mayor o menor concentración de las relaciones de cada sector con el resto y elegimos como criterios de selección un índice de atracción y uno de difusión (inverso del índice relativo de concentración sectorial) superior al nivel promedio, los sectores clave se presentan en el cuadro 4. Es decir, son sectores clave si se pretende incidir no sólo en el volumen de producción nacional, sino también, conseguir una difusión global de las acciones más generalizada.

Es importante observar que utilizando los criterios de atracción y difusión señalados desaparecen sectores como dirección de corporativos (13), comunicación e información en medios (9) e industria de la manufactura (5). Es decir, la economía está especializada en servicios.

Cuadro 4.

Sectores con índices de atracción y difusión superiores al promedio

\begin{tabular}{|l|c|}
\hline \multicolumn{1}{c|}{ Vía ventas } & Vía compras \\
\hline s14. Servicios de apoyo a negocios & s6. Comercio \\
s12. Servicios profesionales & s4. Construcción \\
s3. Electricidad, agua y gas & s7. Transportes \\
s10. Servicios financieros y de seguros & \\
s8. Correos y almacenamiento & \\
\hline
\end{tabular}

Fuente: Elaboración propia. 


\section{Conclusiones}

La búsqueda de sectores clave de la economía basada en relaciones de causalidad (encadenamientos) hacia adelante y hacia atrás ha sido un tópico al que se ha dedicado un amplio esfuerzo intelectual durante las seis últimas décadas.

Sin embargo, surge un problema cuando los sectores clave no se definen exclusivamente en términos del peso económico de los sectores o de la intensidad de las relaciones económicas directas, sino en función del papel que juegan las interrelaciones productivas en el entramado económico o relaciones económicas globales. En ese caso, las relaciones entre diferentes producciones sectoriales son completamente interdependientes. La fuerza y estabilidad de una estructura no sólo depende del peso de las relaciones entre los sectores sino de la posición relativa de los mismos. En este sentido, los grafos valuados y, en concreto, los de influencia relativa, posibilitan realizar este análisis.

En el caso de la economía mexicana, haciendo uso del grafo de influencia (valuado) asociado al modelo de insumo producto, se puede decir identificar como grupo de sectores clave a: la electricidad, agua y gas (3), construcción (4), comercio (6), transportes (7), correo y servicios de almacenamiento (8), servicios financieros y de seguros (10), servicios profesionales (12), dirección de corporativos (13) y servicios de apoyo a negocios (14).

Es importante destacar que los sectores de la industria manufacturera (5), comunicación e información en medios (9) y dirección de corporativos (13) desaparecen del grupo de sectores clave. En lo que se refiere a las ventas, el motivo es que los sectores (9) y (13) dirigen su producción fundamentalmente a la demanda final y no a la demanda intermedia o intersectorial de la economía. Mientras que por el lado de las compras, la ausencia del sector (5), se explica por la elevada concentración de los efectos de difusión hacia los demás sectores del conjunto de la economía.

Por último, debemos advertir que, en términos de su contenido conceptual, esta clasificación sectorial es similar a la utilizada en el enfoque clásico. Es decir, existe correspondencia conceptual entre los sectores estratégicos, independientes, base y clave; sin embargo, difiere el reparto subyacente. Esto se debe a la posición de centralidad de cada sector en la red económica, que les permite transmitir unos efectos totales sobre la economía con relativa rapidez o lentitud, jugando un papel importante o no en la intermediación de las relaciones intersectoriales del resto de la economía. 


\section{Bibliografía}

Aroche-Reyes, Fidel, "Desintegración en la estructura productiva mexicana y el empleo. Los coeficientes importantes y la integración”, en Revista asturiana de economía, núm. 33, 2005, pp. 31-61.

Cella, Guido, "The input-output measurement of inter industry linkages", en Oxford Bulletin of Economics and Statistics, vol. 46. núm. 1, 1984, pp. 73-84.

Chenery, Hollis B. y Watanabe Tsunehiko, "International comparisons of the structure of productions" en Econometrica, vol. 26, núm. 4, 1958, pp. 487-521.

Dirtzenbacher, E. y J. A. Van Der Linden, "Sectorial and spatial linkages in the EC production structure", en Journal of Regional Science, vol. 37, núm. 2, 1997, pp. 235-257.

Fuentes, Noé Arón y Miran Sastré, "Evaluación de la congruencia entre economía y gobierno en torno al desarrollo regional de Baja California Sur, México", en Problemas del desarrollo, vol. 32, núm. 126, México, IIE-UNAM, julio-septiembre de 2001, pp. 149-174.

García, Ana Salomé, y Carmen Ramos, "Core/ periphery structure models: an alternative methodological proposal", en Social Networks, vol. 28, núm. 4, 2006, pp. 442-448. "Las redes sociales como herramienta de análisis estructural input-output", en REDES. Revista hispana para el análisis de redes sociales, vol. 4 , núm. 5 , junio-julio de 2003, http:/ /revista-redes.rediris.es. , Análisis del cambio estructural a partir de un método de extracción, Asturias, Departamento de Economía Aplicada, Universidad de Oviedo, 2002.

García, Ana Salomé, Carmen Ramos y Antonio Morillas Raya, "Relaciones interindustriales y difusión de la innovación. Una aproximación desde la teoría de redes", en Estadística española, vol. 47, núm. 160, 2005, pp. 475-500.

García, María Emilia y María Elisa Amo, “Análisis pretopológico de las relaciones de interdependencia en el modelo input-output de Leontief", VIII Jornadas de ASEPUMA. Sevilla, 2000.

, Modelo Input-Output de Leontief: Teoría de Grafos frente a estructuras pretopológicas, Departamento de Economía y Empresa, Universidad de Castilla-La Mancha, 2000.

Vol. 40, núm. 158, julio-septiembre / 2009
Ghosh, A., "Input-Output approach in an allocation system" en Economica, núm. 25, 1958, pp. 58-64.

Hazari B. R., "Empirical evidence of key sectors in the Indian economy" en Review of Economics and Statistics, vol. 20, 1976, pp. 301-305.

Hirschman, A.O., The Strategy of Economic Development, New Haven, Yale University Press, 1958.

INEGI, http://www.inegi.gob.mx/inegi/default... 2008.

Kozikowski, Z., Técnicas de planeación macroeconómica, Trillas, México, 1988.

Lantner, R., Théorie de la Dominance économique, París, Dunod, 1974.

Morillas, Antonio, "Cambios en la estructura productiva española, 1980-1995. Un análisis estructural mediante la teoría de grafos”, en Miguel Ángel Tarancón Morán y Carmen Ramos Carvajal (coords.), Estructura Input-Output y dinámica económica, San Vicente, Alicante, Editorial Club Universitario, 2004.

"Aplicación de la teoría de grafos al estudio de los cambios en las relaciones intersectoriales de la economía andaluza en la década de los 80", en Cuentas regionales e Input-Output, Instituto de Estadística de Andalucía, 1995.

, La teoría de grafos en el análisis Input-Output. La estructura productiva andaluza, Málaga, Editorial Universidad de Málaga, 1983.

Ponsard C. (ed). "Graphes de transfert et analyse économique”, París, Sirey, 1972.

Rasmussen, P.N., Studies in Intersectorial Relations, Ámsterdam, North-Holland, 1956.

Soza, Sergio, "Análisis estructural de extracción y su comparación con los métodos clásicos de análisis: una aplicación empírica”, en Miguel Ángel Tarancón Morán y Carmen Ramos Carvajal (coords.), Estructura Input-Output y dinámica económica, San Vicente, Alicante, Editorial Club Universitario, 2004.

Strassert, G., "Zur Bestimmuny Strategisher Sektoren mit Hilfe von Input-Output Modellen", en Jahrbücher für Nationalökonomie and Statistik, Stuttgart, núm. 182(3), 1968, pp. 211-215.

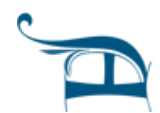

